\title{
Adding Value of Chest CT Images to a Personalized Prognostic Model in Acute Respiratory Distress Syndrome: A Retrospective Study
}

\section{Yuan-Cheng Wang}

Southeast University Zhongda Hospital

\section{Shan Huang}

Southeast University Zhongda Hospital

Qian Yu

Southeast University Zhongda Hospital

\section{Xiangpan Meng}

Southeast University Zhongda Hospital

\section{Jianfeng Xie}

Southeast University Zhongda Hospital

Yi Yang

Southeast University Zhongda Hospital

Shenghong Ju ( $\sim$ jsh@seu.edu.cn )

Southeast University Zhongda Hospital

\section{Research}

Keywords: acute respiratory distress syndrome, radiomics, mechanical ventilation, prediction model

Posted Date: September 13th, 2021

DOl: https://doi.org/10.21203/rs.3.rs-871622/v1

License: (9) This work is licensed under a Creative Commons Attribution 4.0 International License. Read Full License 


\section{Abstract}

Background: Acute respiratory distress syndrome (ARDS) is a critical disease in the intensive care unit (ICU) with high morbidity and mortality. The accuracy for predicting outcomes of ARDS patients with mechanical ventilation is limited, and most predicting model are based on clinical information. This study aimed to explore the prognostic value of chest CT images in ARDS patients with mechanical ventilation and develop an outperformed predictive model.

Methods: The patients diagnosed with ARDS between January 2014 and June 2019 were retrospectively recruited. Clinical information, ventilation parameters, primary causes, illness severity, and chest CT images were collected. Radiomics features were extracted from the levels of the upper, middle, and lower lungs, and were further screened according to the primary outcome (28-day mortality after ARDS onset). Radiomics Scores for each level were computed. The univariate and multivariate logistic regression analyses were applied to figure out risk factors. Various predictive models were constructed and compared.

Results: Of 366 ARDS patients recruited in this study, 276 (median age, 64 years [interquartile range, 5475 years]; 208 male) survive on the Day 28. Among all factors, the APACHE $\otimes$ Score $(\mathrm{OR}, 2.607,95 \% \mathrm{Cl}$ : 1.896-3.584, $P<0.001)$, the Radiomics_Score of the middle lung $(\mathrm{OR}, 2.230,95 \% \mathrm{Cl}: 1.387-3.583, P=$ $0.01)$, the Radiomics_Score of the lower lung (OR, 1.633, 95\% Cl: $1.143-2.333, P=0.01)$ was associated with the 28-day mortality. The clinical_radiomics predictive model (AUC, $0.813,95 \% \mathrm{Cl}$ : $0.767-0.850$ ) show the best performance compared with the clinical model (AUC, $0.758,95 \% \mathrm{Cl}: 0.710-0.802$ ), the radiomics model (AUC, 0.692, 95\% Cl: 0.641-0.739) and the various ventilator parameter-based models (highest AUC, $0.773,95 \% \mathrm{Cl}: 0.726-0.815)$.

Conclusions: The radiomics features of chest CT images have incremental values in predicting the 28day mortality in ARDS patients with mechanical ventilation. These results help to build a personalized prognostic prediction model and to stratify high-risk patients.

\section{Introduction}

Acute respiratory distress syndrome (ARDS), characterized as refractory hypoxemia and respiratory distress, is a severe state of acute lung injury. It may lead to severe respiratory disorders, e.g., coronavirus disease 2019, severe acute respiratory syndrome, and Middle East respiratory syndrome[1]. In the intensive care unit (ICU), $10.4 \%$ of patients suffer ARDS attacks, accounting for $23.4 \%$ of patients needing mechanical ventilation, with mortality between $34.9 \%$ and $46.1 \%[2]$.

Mechanical ventilation is an essential method in the treatment of ARDS[3]. However, some patients do not benefit from this treatment[4]. Researchers have been dedicating to seek indicators for predicting mortality to guide individualized decision-making $[5,6]$. In previous studies, several ventilator parameters, including tidal Volume (Tv)[7], positive end-expiratory pressure (PEEP) [8], plateau pressure and driving pressure[9], and peak inspiratory pressure (PIP)[10], have been proven to be associated with the outcome 
of ARDS. The mechanical power (MP), an integrated parameter computed by several critical variables, has been proven to be a new marker to assess the severity of ARDS[11]. Several models have been developed, but the area under the receiver operating characteristic curve (AUC) is less than satisfactory (approximately 0.75 )[12], better prognostic indicators are urgently needed.

$\mathrm{CT}$ is a vital tool in the evaluation of ARDS. The findings include ground-glass opacifications combined with bilateral consolidation[13,14]. It has been used previously to evaluate mechanical ventilation[15] on patient outcomes [16]. Traditional visual CT interpretation is subjective and less informative. Radiomics, a high-throughput method to extract a large amount of quantitative information from radiographs, has brought massive power to the field of medical imaging[17, 18]. However, no applications in ARDS have been reported.

Therefore, this study aimed to extract radiomics features from chest CT images to develop and validate a prediction model for the 28-day mortality in ARDS patients with mechanical ventilation and compare its performance with previously reported models derived from clinical and ventilator parameters.

\section{Methods}

This was a retrospective study, and the patients' informed consents were waived. The Institutional Ethics Committees approved the study for Clinical Research of the Hospital (No. 2021ZDSYLL060-P01). All patients diagnosed with ARDS between January 2014 and June 2019 in a tertiary ICU of a university hospital were primarily considered. The diagnostic criteria of ARDS were according to the Berlin definition[1], proposed in 2012. Further inclusion and exclusion procedures were conducted. The study flowchart is provided in Fig. 1.

Inclusion criteria: 1) treated with mechanical ventilation; 2) underwent chest CT 24 hours before or after mechanical ventilation.

Exclusion criteria: 1) Inadequate CT image quality; 2) Patients under 18 years old; 3) Patients with underlying pulmonary diseases including chronic obstructive pulmonary disease, tuberculosis, asthma, pulmonary fibrosis, and pulmonary tumor or with the history of pulmonary lobectomy.

The following patients' data were collected: 1) Demographics: age, gender, height, weight, Acute Physiologic and Chronic Health Evaluation (APACHE) $\otimes$ score; 2) Initial (day 0) mechanical ventilator parameters: $\mathrm{PaO}_{2} / \mathrm{FiO}_{2}$ ratio (P/F ratio), Tv, PEEP, PIP, respiratory rate (RR).

The primary outcome was the all-cause mortality on Day 28 after ARDS onset. Patients discharged in 28 days were followed up via telephone at day 28 , and the survival status was recorded.

The secondary outcome was the all-cause mortality on Day 10 after ICU admission, the ICU length of stay, and the hospitalization length of stay.

CT acquisition 
All recruited patients underwent non-contrast chest CT examinations in a supine position within 24 hours before or after ICU admission. All CT examinations were performed with one of the following CT scanners: Discovery CT750 HD (GE MEDICAL SYSTEMS), Optima CT670 (GE MEDICAL SYSTEMS), Revolution CT (GE MEDICAL SYSTEMS), SOMATOM Sensation 64 (SIEMENS). The main parameters for scanning were as follows: tube-voltage 100-140 kVp, tube-current 150-190 mAs, matrix $512 \times 512$. The images were exported and saved in Digital Imaging and Communication in Medicine (DICOM) format.

Image analysis

Three regions of interest (ROIs) were delineated manually at the lung's upper, middle, and lower levels and were saved as label_1, label_2, and label_3 separately. The upper, middle and lower levels were defined as $2 \mathrm{~cm}$ above the carina, $1 \mathrm{~cm}$ below the carina, and $1 \mathrm{~cm}$ above the right hemidiaphragm. Adjustment in some patients was allowed due to varied body sizes. All these ROIs were drawn in ITK-SNAP version 3.8 (www.itksnap.org/). An example was demonstrated in Fig. 2.

The radiomics features were extracted in Python 3.7 with the package of Pyradiomics 3.0.0 (https://pyradiomics.readthedocs.io/en/latest/). A total of 1218 features of each label including First Order, Shape, Gray Level Co-occurrence Matrix (GLCM), Gray Level Size Zone Matrix (GLSZM), Gray Level Run Length Matrix (GLRLM), Neighboring Gray Tone Difference Matrix (NGTDM), Gray Level Dependence Matrix (GLDM) were extracted. These features were extracted from the original and the derived images by applying Laplacian of Gaussian filtering or Wavelet filtering.

First of all, the Synthetic Minority Oversampling TEchnique (SMOTE) was applied to balance the positive/negative samples. Normalization and standardization were then applied to the feature matrix. The data were separated into the training set and the validation set with a proportion of approximately 2:1. The validation set was fixed in follow-up work. Pearson Correlation Coefficients were used to achieve dimensional reduction, and analysis of variance (ANOVA) was used to evaluate the relationship between features and the outcome. The logistic regression analysis was then performed to build radiomics signatures for the each label. Radiomics scores were calculated and recorded as Radiomics_Score_1, Radiomics_Score_2, Radiomics_Score_3 for the three levels, relatively. All these statistics were performed in Feature Analysis Explorer (FAE, https://github.com/salan668/FAE)[19].

\section{Statistical Analysis}

The cohort was divided into survivors and non-survivors based on the outcome on Day 28. The continuous variables were presented as means (standard deviations) or medians (interquartile ranges [IQR]) by group and were compared with student's t-test or Mann-Whitney U test. The categorical variables were expressed as frequency and percentage and were compared with the Chi-square test or Fisher's exact test.

All continuous variables were normalized, and the univariate and multivariate logistic regression analysis were applied to explore risk factors. Odds ratios (ORs) and 95\% confidence intervals (Cls) were 
calculated. The 2-tailed $P<0.05$ was considered statistically significant. Various models for predicting 28day mortality were constructed. The clinical model included APACHE $₫$ score. The radiomics model

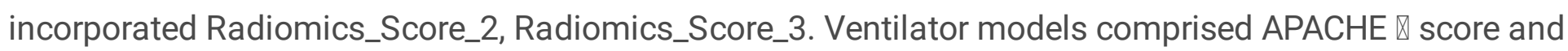
each ventilator parameter, respectively. These ventilator parameters were Tv, tidal Volume normalized to predicted body weight (Tv/PBW), RR, PIP, PEEP, driving pressure, MP, MP normalized to predicted body weight (MP/PBW), MP normalized to compliance (MP/compliance). The predictive performance of these models was evaluated by AUC and compared by Delong's test. All these statistics were performed in MedCalc version 19.7.4.

Formulas related to ventilator parameters calculation were as follows:

Driving pressure $\triangle P=P I P-P E E P$;

$\mathrm{MP}(\mathrm{J} / \mathrm{min})=0.098 \times \mathrm{Tv} \times \mathrm{RR} \times(\mathrm{PIP}-\triangle \mathrm{P} \times 0.5) ;$

\section{Results}

A total of 366 patients (276 survivors and 90 non-survivors) were finally included in this study. Characteristics of included patients were presented in Table 1 . Age and sex were equally distributed ( $P=$ $0.43, P=0.97)$ in two groups. The APACHE $\square$ score in non-survivors (30 [23-35]) was higher than that in survivors $(21$ [16-27], $P<0.001)$. The ICU length of stay and hospitalization showed no difference between the two groups $(P=0.29, P=0.37)$. For primary causes of ARDS, most patients were due to intrapulmonary factors ( $88.0 \%$ in survivors and $90.0 \%$ in non-survivors). The number of patients without sepsis was slightly higher than those with sepsis but with no statistical significance $(P=0.18)$. The percentage of patients with moderate severity was highest in all severity subgroups $(51.1 \%$ in survivors and $47.8 \%$ in non-survivors). 
Table 1

Baseline Characteristics of the included patients

\begin{tabular}{|llll|}
\hline Characteristics & Survivors(n= 276) & Non-survivors(n= 90) & P value \\
\hline Age $(\mathrm{y})^{*}$ & $64(50-75)$ & $66.5(51-78)$ & 0.43 \\
\hline Sex, male, $\%$ & $208(75.4 \%)$ & $68(75.6 \%)$ & 0.97 \\
\hline Primary cause of ARDS & & & 0.61 \\
\hline Intra-pulmonary & $243(88.0 \%)$ & $81(90.0 \%)$ & \\
\hline Extra-pulmonary & $33(12.0 \%)$ & $9(10.0 \%)$ & \\
\hline ARDS Severity & & $12(13.3 \%)$ & \\
\hline Mild & $65(23.6 \%)$ & $43(47.8 \%)$ & 0.18 \\
\hline Moderate & $141(51.1 \%)$ & $35(38.9 \%)$ & \\
\hline Severe & $70(25.3 \%)$ & & $<0.001$ \\
\hline Sepsis & $117(42.4 \%)$ & $31(34.4 \%)$ & 0.29 \\
\hline With & $159(57.6 \%)$ & $30(65.6 \%)$ & 0.37 \\
\hline Without & $21(16-27)$ & $12(8-20)$ & \\
\hline APACHE $\otimes$ Score* & $11(5-21)$ & $18(9-32)$ & \\
\hline Length of ICU stay* & $17(8-28)$ & & \\
\hline Length of hospitalization* & & & \\
\hline Note. - Unless otherwise indicated, data are numbers of patients, with percentages in parentheses. & \\
\hline *Data are medians, with interquartile ranges in parentheses. & & \\
\hline
\end{tabular}

A total of 1218 features of each label was extracted. Seven features at the upper lung level were selected with most characteristics related to texture uniformity. At the middle lung level, twelve features were selected, most of which related to density distribution. As for the lower lung, only three features, including Interquartile Range, Mean, and the Median after wavelet transformation, were selected. Detailed information about selected radiomics features was listed in the Supplemental material Table S1. More explanation of the radiomics features can refer to the website (https://pyradiomics.readthedocs.io/en/latest/features.html).

The P/F ratio $(P=0.003)$, APACHE $\square$ score $(P<0.001)$, Radiomics_Score_1 $(P<0.001)$, Radiomics_Score_2 $(P<0.001)$, Radiomics_Score_3 $(P<0.001)$, Tv/PBW $(P=0.03), \operatorname{RR}(P=0.03), \mathrm{MP}(P=0.01)$ and MP/PBW $(P=0.01)$ were associated with the 28-Day mortality in the univariate logistic regression analysis (Table 2). After the multivariate analysis, three independent risk factors including APACHE $\triangle$ score (OR, 
2.607, 95\% Cl: $1.896-3.584, P<0.001)$, Radiomics_Score_2 (OR, 2.230, 95\% Cl: $1.387-3.583, P=0.01)$, Radiomics_Score_3 (OR, 1.633, 95\% Cl: $(1.143-2.333, P=0.01)$ were selected at last. 
Table 2

Factors associated with 28-Day mortality in ARDS patients with mechanical ventilation.

Univariate analysis

Multivariate analysis

\begin{tabular}{|c|c|c|c|c|}
\hline Variables & Estimate $(95 \% \mathrm{Cl})$ & P-value & Estimate $(95 \% \mathrm{Cl})$ & P-value \\
\hline Age (y) & $\begin{array}{l}0.999(0.985- \\
1.012)\end{array}$ & 0.86 & NS & NS \\
\hline Sex (male) & $\begin{array}{l}1.119(0.635- \\
1.973)\end{array}$ & 0.70 & NS & NS \\
\hline Primary causes of ARDS & $\begin{array}{l}1.029(0.470- \\
2.251)\end{array}$ & 0.94 & NS & NS \\
\hline \multicolumn{5}{|l|}{ Severity of ARDS } \\
\hline Moderate & $\begin{array}{l}1.363(0.668- \\
2.783)\end{array}$ & 0.39 & NS & NS \\
\hline Severe & $\begin{array}{l}2.269(1.077- \\
4.780)\end{array}$ & 0.03 & NS & NS \\
\hline With/without sepsis & $\begin{array}{l}1.439(0.856- \\
2.419)\end{array}$ & 0.16 & NS & NS \\
\hline $\mathrm{P} / \mathrm{F}$ ratio & $\begin{array}{l}0.609(0.440- \\
0.884)\end{array}$ & 0.003 & NS & NS \\
\hline APACHE $\otimes$ Score & $\begin{array}{l}2.798(2.058- \\
3.805)\end{array}$ & $<0.001$ & $2.607(1.896-3.584)$ & $<0.001$ \\
\hline Radiomics_Score_1 & $\begin{array}{l}1.762(1.325- \\
2.343)\end{array}$ & $<0.001$ & NS & NS \\
\hline Radiomics_Score_2 & $\begin{array}{l}2.621(1.678- \\
4.093)\end{array}$ & $<0.001$ & $2.230(1.387-3.583)$ & 0.01 \\
\hline Radiomics_Score_3 & $1.978(1.395-2.805)$ & $<0.001$ & $1.633(1.143-2.333)$ & 0.01 \\
\hline Tidal volume & $\begin{array}{l}1.251(0.985- \\
1.590)\end{array}$ & 0.06 & NS & NS \\
\hline Tidal volume/PBW & $\begin{array}{l}1.299(1.020- \\
1.654)\end{array}$ & 0.03 & NS & NS \\
\hline Respiratory Rate & $\begin{array}{l}1.310(1.032- \\
1.664)\end{array}$ & 0.03 & NS & NS \\
\hline $\begin{array}{l}\text { Positive End-Expiratory } \\
\text { Pressure }\end{array}$ & $\begin{array}{l}1.177(0.927- \\
1.493)\end{array}$ & 0.18 & NS & NS \\
\hline Peak Inspiratory Pressure & $\begin{array}{l}1.080(0.846- \\
1.379)\end{array}$ & 0.54 & NS & NS \\
\hline Driving_pressure & $\begin{array}{l}0.982(0.766- \\
1.259)\end{array}$ & 0.88 & NS & NS \\
\hline
\end{tabular}




\section{Univariate analysis}

Mechanical power

Mechanical power /PBW

$1.363(1.083-$

1.715)

$1.397(1.109-$

1.761)

\section{Multivariate analysis}

0.01 NS NS

$0.01 \quad N S$

NS

NS = Not significant; $\mathrm{PBW}=$ Predicted body weight $; \mathrm{Cl}=$ Confidence interval .

A clinical model (APACHE $\triangle$ score), a radiomics model (Radiomics_Score_2 + Radiomics_Score_3), and a clinical_radiomics model (APACHE $\otimes$ score + Radiomics_Score_2 + Radiomics_Score_3) to predict the 28Day mortality were constructed. The AUC of these three models in validation set were $0.758(95 \% \mathrm{Cl}$ : 0.710 to 0.802$), 0.692$ ( $95 \% \mathrm{Cl}: 0.641$ to 0.739$), 0.813$ (95\% Cl: 0.767 to 0.850 ) respectively (Fig. 3 ). The difference between the clinical model and the radiomics model showed no significance $(P=0.13$, Delong test). When combined together, the clinical_radiomics model demonstrated higher predictive power than clinical model $(P=0.004)$ as well as the radiomics model $(P<0.001)$. Figure 3 shows the ROC curve of the three models in the training set and validation set. For the clinical_radiomics model, the sensitivity and specificity were $92.5 \%$ and $58.7 \%$, respectively.

A total clinical_radiomics score was computed by the coefficient in the final logistic regression clinical_radiomics model. A cutoff value was defined as the corresponding score when maximum value of sensitivity plus specificity was achieved. The change of sensitivity and specificity with the clinical_radiomics score and the distribution of clinical_radiomics score in two groups are presented in supplementary material Fig. S1. All cases were divided into the lower-risk and high-risk groups with a cutoff clinical_radiomcis score of 2.3. The Kaplan-Meier survival analysis on Day 28 after ARDS onset is shown in Fig. 4A, with the log-rank test $(P<0.001)$.

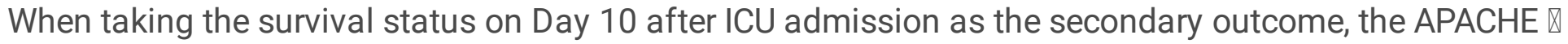
score, Radiomics_Score_2, Radiomics_Score_3 also showed statistical significance.

The odds ratios of these factors were APACHE $₫$ score (OR, $1.108,95 \% \mathrm{Cl}: 1.067$ to $1.149, P<0.001)$, Radiomics_Score_2 (OR, 1.906, 95\% Cl: 1.044 to 3.478, $P=0.03)$, Radiomics_Score_3 (OR, $1.665,95 \% \mathrm{Cl}$ : 1.189 to $2.332, P=0.003$ ). The AUC was 0.791 (95\% Cl: 0.746 to 0.831 ), sensitivity was $76.8 \%$ and specificity was $71.0 \%$.

With the cutoff clinical_radiomics score of 2.3, the high-risk and low-risk groups also showed significant difference by Kaplan-Meier survival analysis, shown in Fig. 4B $(P<0.001$, log-rank test).

However, the correlation between these factors and the ICU length of stay or hospitalization was relatively low.

Models constructed by clinical information (APACHE $\triangle$ score) and each ventilator parameter were summarized in Table 3. The Tv and Tv/PBW models were ranked the top two among all models $(0.773$, 
$95 \% \mathrm{Cl}: 0.726$ to 0.815 and $0.770,95 \% \mathrm{Cl}: 0.723$ to 0.812 ). The AUCs of the remaining models were all less than 0.770 .

Table 3

Performance of models in predicting 28-Day mortality

\begin{tabular}{|c|c|c|c|}
\hline & AUC & Lower limit of $95 \% \mathrm{Cl}$ & Upper limit of $95 \% \mathrm{Cl}$ \\
\hline Clinical model & 0.758 & 0.710 & 0.802 \\
\hline \multicolumn{4}{|l|}{ Ventilator parameters models } \\
\hline Tidal Volume(ml) & 0.773 & 0.726 & 0.815 \\
\hline Tidal Volume/PBW(ml/kg) & 0.770 & 0.723 & 0.812 \\
\hline Respiratory rate (/min) & 0.765 & 0.718 & 0.807 \\
\hline Peak Inspiratory Pressure $\left(\mathrm{cmH}_{2} 0\right)$ & 0.762 & 0.715 & 0.805 \\
\hline Positive End-Expiratory Pressure $\left(\mathrm{cmH}_{2} \mathrm{O}\right)$ & 0.762 & 0.715 & 0.805 \\
\hline Driving pressure $\left(\mathrm{cmH}_{2} 0\right)$ & 0.762 & 0.714 & 0.804 \\
\hline Mechanical power (J/min) & 0.769 & 0.722 & 0.811 \\
\hline Mechanical power /PBW $\left(10^{-3} \mathrm{~J} / \mathrm{min} / \mathrm{kg}\right)$ & 0.769 & 0.722 & 0.811 \\
\hline Radiomics model & 0.692 & 0.641 & 0.739 \\
\hline Clinical_Radiomics model & 0.813 & 0.767 & 0.850 \\
\hline \multicolumn{4}{|c|}{$\begin{array}{l}\text { Note. - The clinical model is based on APACHE } \otimes \text { score; The ventilator parameters models include the } \\
\text { APACHE } \ \text { score and each of the individual ventilator parameters. The radiomics model comprises } \\
\text { radiomics_score_2, radiomics_score_3. }\end{array}$} \\
\hline \multicolumn{4}{|c|}{ PBW = Predicted body weight; $\mathrm{AUC}=$ Area under the curve; $\mathrm{Cl}=$ Confidence interval. } \\
\hline
\end{tabular}

When comparing the top two ventilator models with the clinical, radiomics, and clinical_radiomics models, the clinical_radiomcis model showed the best performance $(P=0.02$ with Tv model and $P=0.01$ with Tv/ PBW model).

\section{Discussion}

In critical care medicine, ARDS remains an important life-threatening issue. In a retrospective study[20] of 18 ICUs in mainland China, though ARDS occurs in a low incidence (3.57\%), it results in high in-hospital mortality (46.3\%). The most common risk factors of ARDS attribute to intrapulmonary disorders (83.7\%), of which similar results can be found in our study $(88 \%)$. The proportions of mild, moderate, and severe illness were $9.7 \%, 47.4 \%, 42.9 \%$, respectively, which are also similar to the distribution in our cohort 
(23.6\%, $51.1 \%, 25.3 \%$ in survivors, $13.3 \%, 47.8 \%, 38.9 \%$ in non-survivors). These results could indicate the reliability of the data from this cohort and potentially be comparable.

Among all demographic characteristics and clinical information (age, sex, primary causes of ARDS, the severity of ARDS, with/without sepsis, P/F ratio, APACHE $\triangle$ Score), only the severity of ARDS and the APACHE $\triangle$ Score associated with the mortality in our cohort. The mortality rate increased with the severity, but this effect was eliminated following multivariate regression analysis. The final clinical model in this

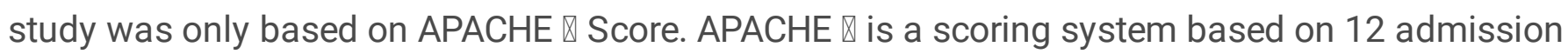
physiologic variables, age and chronic health status. It is a widely used ICU scoring system and has been recognized as a robust prognostic marker for ARDS. Even so, the reported predictive strength is approximately 0.75 evaluated with AUC[21].

Researchers are dedicating to explore other markers aside from APACHE $₫$ scores. A recent paper in Intensive care medicine summarized the predictive models of ventilator parameters, and found favorable predictive value with AUC ranging from 0.743 to $0.753[12]$. Our study has roughly confirmed these results by constructing various ventilator models in our cohort using the same parameters. The AUCs ranged from 0.759 to 0.773 , demonstrating a general consistency. Our study did not show statistical significance between each of the ventilator models, which indicates none of these ventilator models has predominant predictive power over others.

In our study, we integrated APACHE $\triangle$ Score with radiomics and built a new clinical_radiomics model, which demonstrated improved predictive strength compared with clinical model as well as ventilator models. As for the detailed radiomics features selected in the model, most reflect homogeneity and dispersion of pulmonary opacities. For the upper lung, most related features were grouped to the uniformity and the entrophy, while for middle and lower lungs, the features related to the skewness, kurtosis, mean density, et al. in the cluster of first-order contribute more. Up to now, from the pulmonary radiographic change perspective, only morphology phenotypes[22-24], which include the diffused, focal, and patchy classification, and the opacity density or its proportion[14], were reported to be associated with the outcome of ARDS. And this study would potentially be helpful in quantitatively characterize the opacity.

With this clinical_radiomics model, we stratified the patients into high and low-risk groups using a cutoff value and observed a significant difference in mortality between groups. The cutoff value showed a sensitivity of $92.5 \%$, which is high enough for recognizing high-risk patients, and could make earlier personalized intervention possible.

Limitations could also be found in this study. First of all, the method to delineate the ROls could be controversial. We selected three two-dimensional CT sections for evaluation in this study. It cannot be denied that three-dimensional segmentation of the total lesions and the whole lung would give a clearer denotation. Researchers have been contributed to the automatic three-dimensional segmentation of the lung $[25,26]$. However, the accuracy of segmentation is unsatisfactory in the setting of ARDS. The twodimensional CT segmentation is much easier to acquire and more ready to be applied in a clinical setting. 
Secondly, its retrospective nature and selection bias cannot be ignored. Though CT is valid for the evaluation of ARDS and should be done as soon as possible after the onset of the illness, the critical situation of the patients may not allow them to be transported to do the examination. The patients included in this study may not be representative of the whole cohort of ARDS. However, the similar distribution of characteristics with other studies indicates comparability. In addition, the general consistent performance of various ventilator models in our cohort also enhances the study's reliability.

\section{Conclusions}

In conclusion, this study demonstrated that the radiomics information from the chest CT images could add incremental value in predicting ARDS prognosis. A better predictive model was achieved by integrating the APACHE $\triangle$ Score with Radiomics of chest CT images. Most radiomics features selected were related to homogeneity and dispersion. The Middle and lower lung showed more predictive value than the upper lung. Patients with more heterogeneous and more diffused patterns of pulmonary opacity may have a worse prognosis.

\section{Abbreviations}

Acute respiratory distress syndrome

ARDS; Intensive care unit = ICU; Tidal volume $=$ TV; Positive end-expiratory pressure $=$ PEEP; Peak inspiratory pressure $=\mathrm{PIP}$; mechanical power $=\mathrm{MP}$; Area under the receiver operating characteristic curve $=$ AUC; Acute Physiologic and Chronic Health Evaluation = APACHE; $\mathrm{PaO}_{2} / \mathrm{FiO}_{2}=\mathrm{P} / \mathrm{F}$; respiratory rate $=\mathrm{RR}$; Tv/PBW = Tidal volume normalized to predicted body weight;

\section{Declarations}

Ethics approval: This study was approved by the Institutional Ethics Committees for Clinical Research of the Hospital (No. 2021ZDSYLL060-P01).

Consent to participate: The consent was waived.

Consent for publication: Not applicable.

Availability of data and materials: The datasets used and analyzed during the current study are available from the corresponding author on reasonable request

Competing interests: The authors declare that they have no competing interests.

\section{Funding}

This study has received funding from the National Natural Science Foundation of China (NSFC, No. $81801669,81830053)$, and the Natural Science Foundation of Jiangsu Province (BK20180378) 
Author's contributions

WYC, JSH had full access to all of the data in the study and takes responsibility for the integrity of the data and the accuracy of the data analysis, including and especially any adverse effects. WYC, JSH, HS, $Y Q, M X P, X J F, Y Y$ contributed substantially to the study design, data analysis and interpretation. All authors contributed the writing of the manuscript.

\section{Acknowledgements: None}

\section{References}

1. Ranieri VM, Rubenfeld GD, Thompson BT, Ferguson ND, Caldwell E, Fan E, et al. Acute respiratory distress syndrome: The Berlin definition. JAMA. 2012;307:2526-33.

2. Bellani G, Laffey JG, Pham T, Fan E, Brochard L, Esteban A, et al. Epidemiology, patterns of care, and mortality for patients with acute respiratory distress syndrome in intensive care units in 50 countries. JAMA. 2016;315:788-800.

3. Fan E, Brodie D, Slutsky AS. Acute respiratory distress syndrome advances in diagnosis and treatment. JAMA. 2018;319:698-710.

4. Vincent J. Multicenter, randomized, controlled trials evaluating mortality in intensive care: Doomed to fail? Crit Care Med. 2008;36:1311-22.

5. Putensen C, Theuerkauf N. Meta-analysis: Ventilation Strategies and Outcomes of the Acute Respiratory Distress Syndrome and Acute Lung Injury. Ann Intern Med. 2009;151:566-76.

6. Choi W, Shehu E, Lim SY, Koh SO, Na S, Lim C, et al. Markers of poor outcome in patients with acute hypoxemic respiratory failure. J Crit Care. 2014;29:797-802.

7. ARDS Network. Ventilation With Lower Tidal Volumes As Compared With Traditional Tidal Volumes for ALI and the ARDS. N Engl J Med. 2000;342:1301-8.

8. Review S, Meade M, Mercat A, Brower RG, Talmor D, Walter SD, et al. Higher vs Lower Positive EndExpiratory Pressure in Patients With Acute Lung Injury. JAMA. 2015;303:865-73.

9. Amato MBP, Meade MO, Slutsky AS, Brochard L, Costa ELV, Schoenfeld DA, et al. Driving pressure and survival in the acute respiratory distress syndrome. N Engl J Med. 2014;372:747-55.

10. Kao K, Hsieh M, Lin S, Chuang L, Chang C, Hu H, et al. Survival predictors in elderly patients with acute respiratory distress syndrome: a prospective observational cohort study. Sci Rep. 2018;8:1-9.

11. Neto AS, Deliberato RO, Johnson AEW, Bos LD, Amorim P, Pereira SM, et al. Mechanical power of ventilation is associated with mortality in critically ill patients: an analysis of patients in two observational cohorts. Intensive Care Med. 2018;44:1914-22.

12. Zhang Z, Zheng B, Liu N, Ge H, Hong Y. Mechanical power normalized to predicted body weight as a predictor of mortality in patients with acute respiratory distress syndrome. Intensive Care Med. 2019;45:856-64. 
13. Rouby JJ, Puybasset L, Nieszkowska A, Lu Q. Acute respiratory distress syndrome: Lessons from computed tomography of the whole lung. Crit Care Med. 2003;31:285-95.

14. Chung JH, Kradin RL, Greene RE, Shepard J-AO, Digumarthy SR. CT predictors of mortality in pathology confirmed ARDS. Eur Radiol. 2011;21:730-7.

15. Chiumello D, Marino A, Brioni M, Cigada I, Menga F, Colombo A, et al. Lung recruitment assessed by respiratory mechanics and computed tomography in patients with acute respiratory distress syndrome what is the relationship? Am J Respir Crit Care Med. 2016;193:1254-63.

16. Ichikado K, Suga M, Muranaka H, Gushima Y, Miyakawa H, Tsubamoto M, et al. Prediction of Prognosis for Acute Respiratory Distress. 2006;238:321-9.

17. Lambin P, Rios-Velazquez E, Leijenaar R, Carvalho S, Van Stiphout RGPM, Granton P, et al. Radiomics: Extracting more information from medical images using advanced feature analysis. Eur $\mathrm{J}$ Cancer. 2012;48:441-6.

18. Hawkins S, Wang H, Liu Y, Garcia A, Stringfield O, Krewer H, et al. Predicting Malignant Nodules from Screening CT Scans. J Thorac Oncol. 2016;11:2120-8.

19. Song Y, Zhang J, Zhang Y-D, Hou Y, Yan X, Wang Y, et al. FeAture Explorer (FAE): A tool for developing and comparing radiomics models. PLoS One. 2020;15:e0237587.

20. Huang X, Zhang R, Fan G, Wu D, Lu H, Wang D, et al. Incidence and outcomes of acute respiratory distress syndrome in intensive care units of mainland China: a multicentre prospective longitudinal study. Crit Care. 2020;24:515.

21. Rahmatinejad Z, Tohidinezhad F, Reihani H, Rahmatinejad F, Pourmand A, Abu-Hanna A, et al. Prognostic utilization of models based on the APACHE II, APACHE IV, and SAPS II scores for predicting in-hospital mortality in emergency department. Am J Emerg Med. 2020;38:1841-6.

22. Rouby JJ, Puybasset L, Cluzel P, Richecoeur J, Lu Q, Grenier P. Regional distribution of gas and tissue in acute respiratory distress syndrome. II. Physiological correlations and definition of an ARDS Severity Score. CT Scan ARDS Study Group. Intensive Care Med. 2000;26:1046-56.

23. Puybasset L, Gusman P, Muller JC, Cluzel P, Coriat P, Rouby JJ. Regional distribution of gas and tissue in acute respiratory distress syndrome. III. Consequences for the effects of positive endexpiratory pressure. CT Scan ARDS Study Group. Adult Respiratory Distress Syndrome. Intensive Care Med. 2000;26:1215-27.

24. Puybasset L, Cluzel P, Gusman P, Grenier P, Preteux F, Rouby JJ. Regional distribution of gas and tissue in acute respiratory distress syndrome. I. Consequences for lung morphology. CT Scan ARDS Study Group. Intensive Care Med. 2000;26:857-69.

25. Gill G, Beichel RR. An approach for reducing the error rate in automated lung segmentation. Comput Biol Med. 2016;76:143-53.

26. Klapsing P, Herrmann P, Quintel M, Moerer O. Automatic quantitative computed tomography segmentation and analysis of aerated lung volumes in acute respiratory distress syndrome-A comparative diagnostic study. J Crit Care. 2017;42:184-91. 
Figures

Patients diagnosed with ARDS from 2014.01 to 2019.06

Inclusion criteria

$\checkmark$ Treated with mechanical ventilation

$\checkmark$ Underwent chest CT in $\mathbf{2 4}$ hours before or after mechanical ventilation

Patients preliminary included $(\mathrm{N}=474)$

Exclusion criteria

$\checkmark$ Patients under 18 years old $(\mathrm{N}=4)$

$\checkmark$ Inadequate $C T$ image quality $(N=11)$

$\checkmark$ Patients with pulmonary diseases* $(N=93)$

Patients finally included $(\mathrm{N}=366)$

Survival $(N=276) \&$ Non-survival $(N=90)$

Training set $(\mathrm{N}=\mathbf{2 5 6})$

Validation set $(\mathrm{N}=110)$

Figure 1

Study flowchart. 


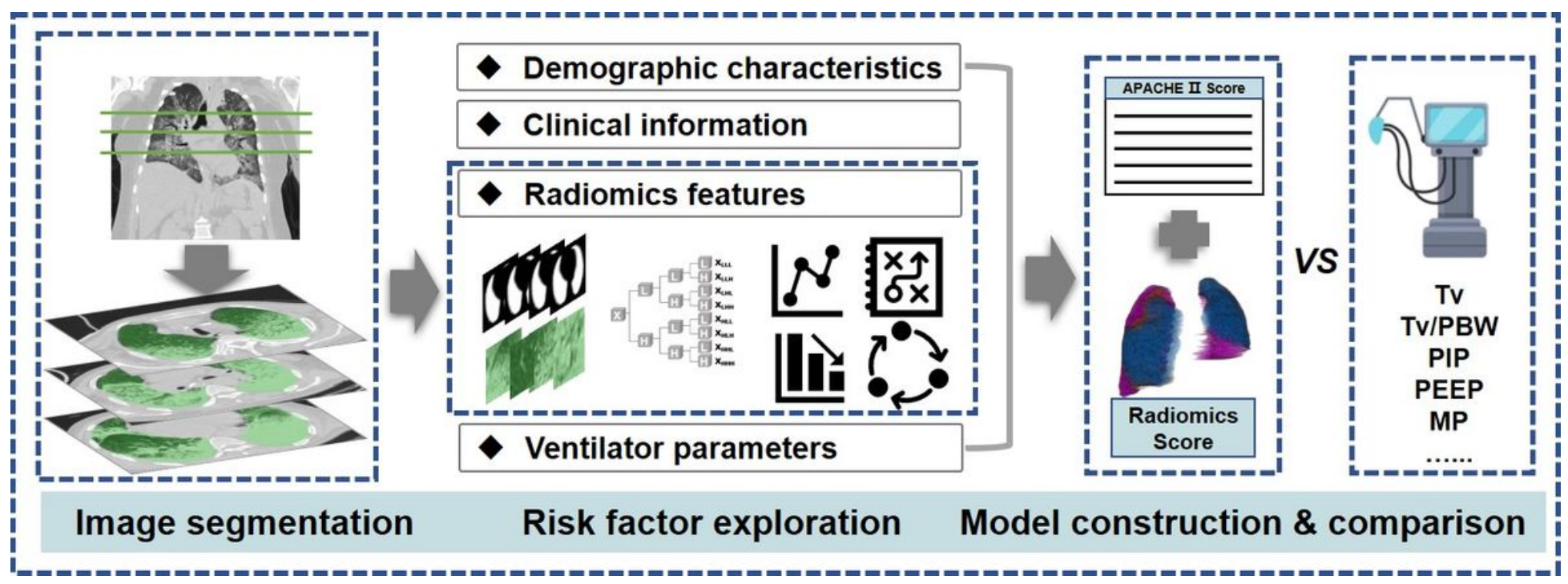

Figure 2

Schematic diagram of the study.
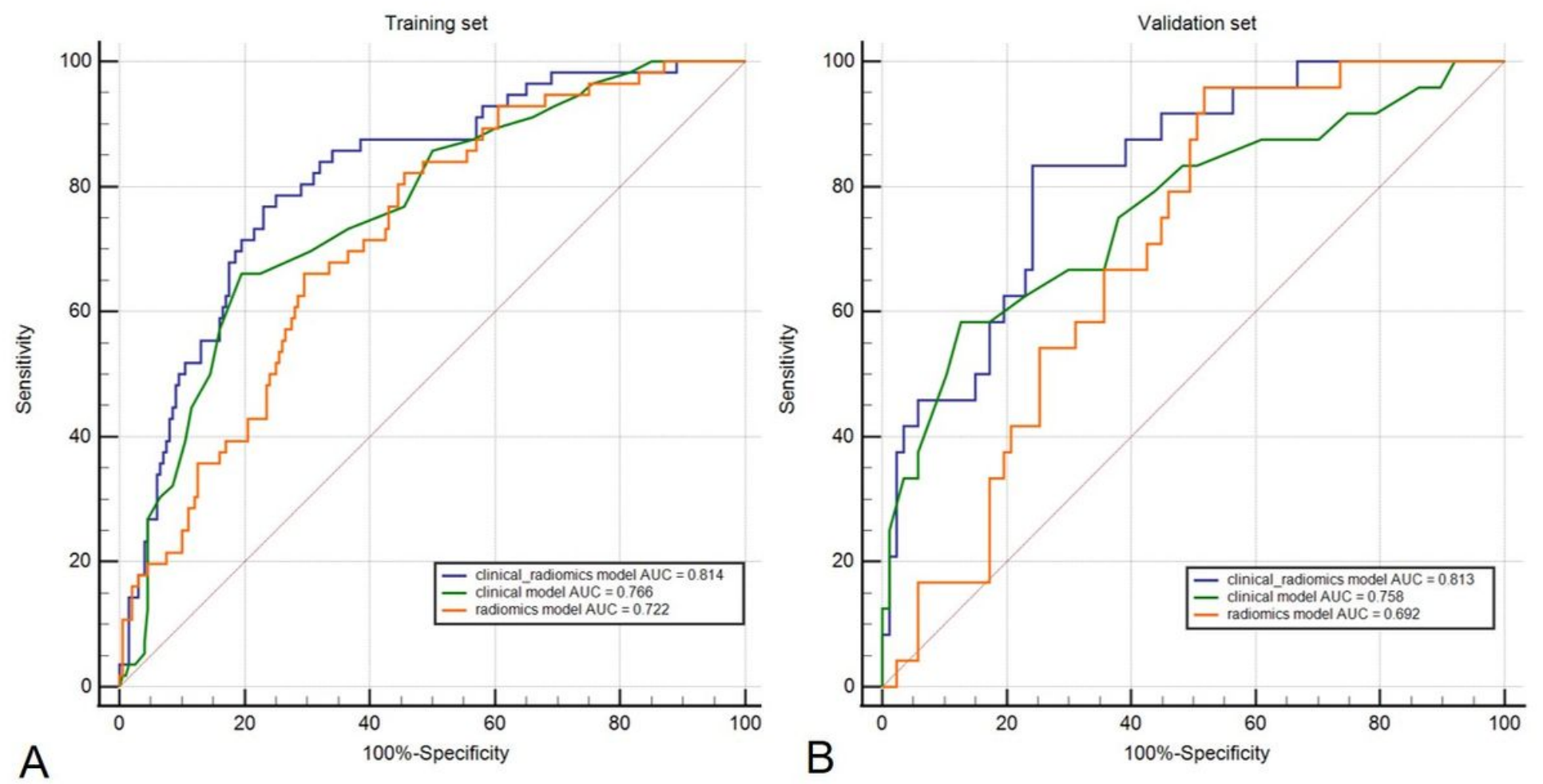

Figure 3

The ROC curve of the radiomics model, clinical model, and clinical_radiomics model in the training and validation sets. 


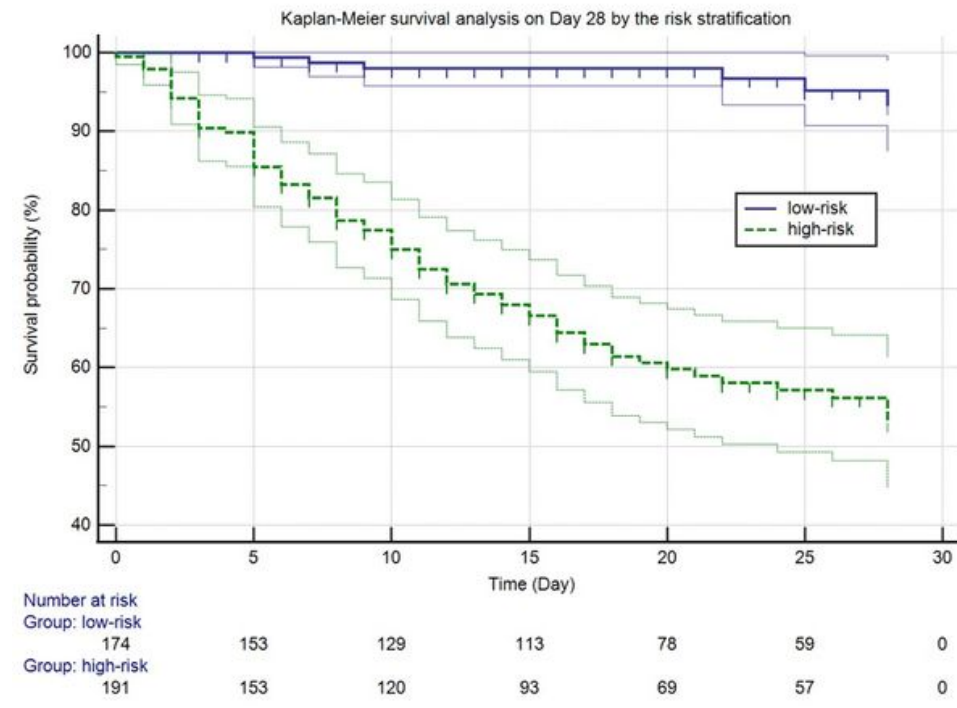

A

\section{Figure 4}

The Kaplan-Meier survival analysis of the 28-Day mortality after ARDS onset and 10-Day mortality after ICU admission by risk stratification.

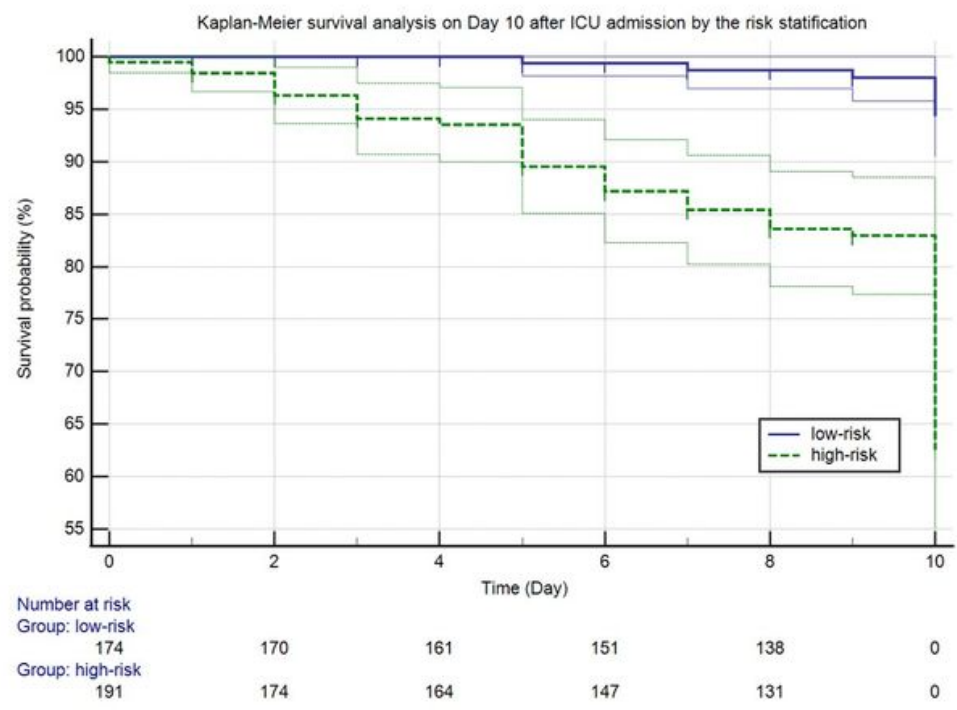

B 


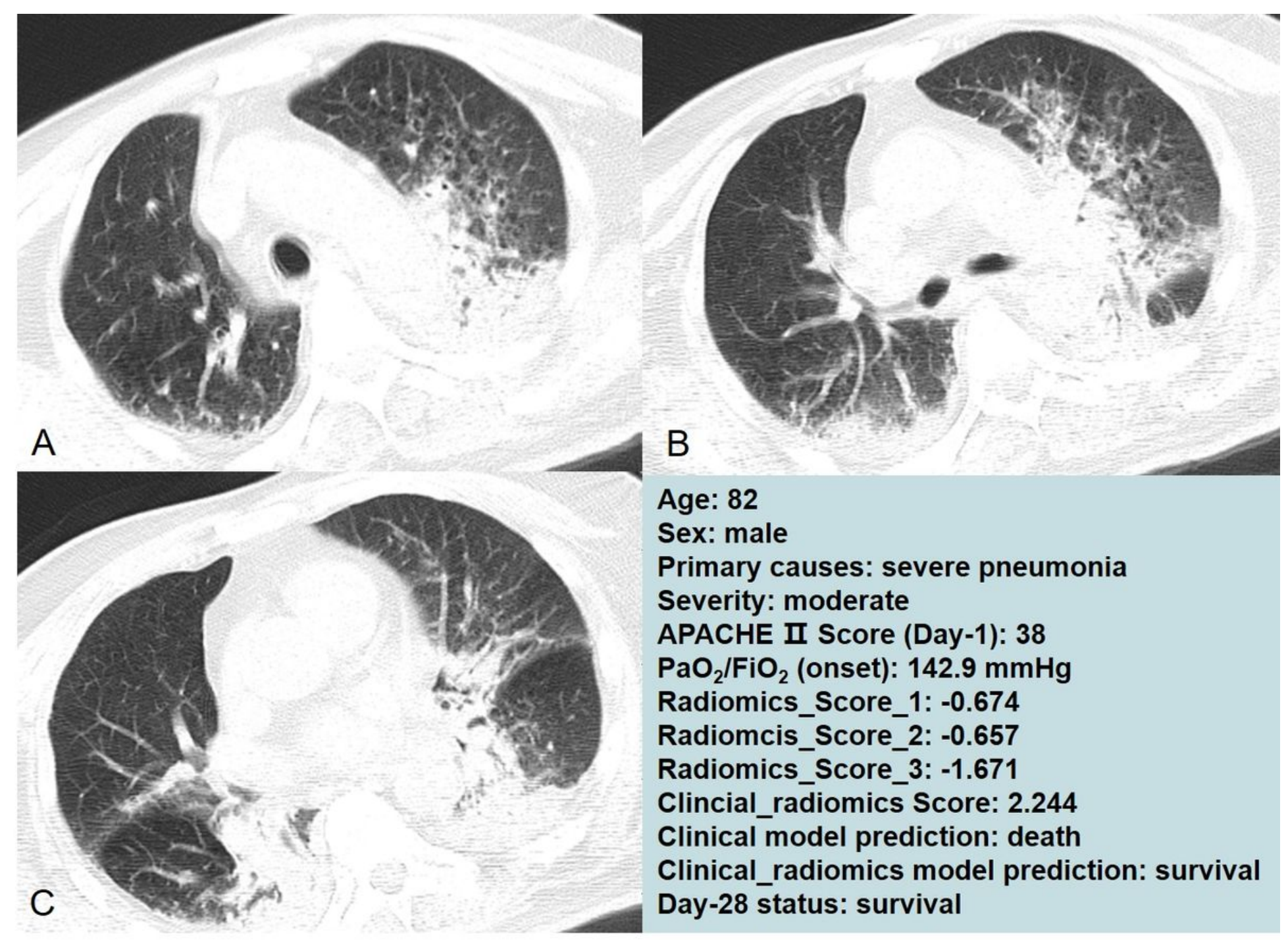

Figure 5

Chest CT images of an 82-year-old male admitted to the intensive care unit for acute respiratory distress syndrome with no special previous history. The CT images were obtained within 24 hours of illness onset. The upper (A), middle (B), and lower (C) lungs show bilateral focal opacity, especially in the posterior and inferior of the lung. The table show specific metrics. The male survived after 28 days of illness onset, and the clinical_radiomics model made the correct prediction. 


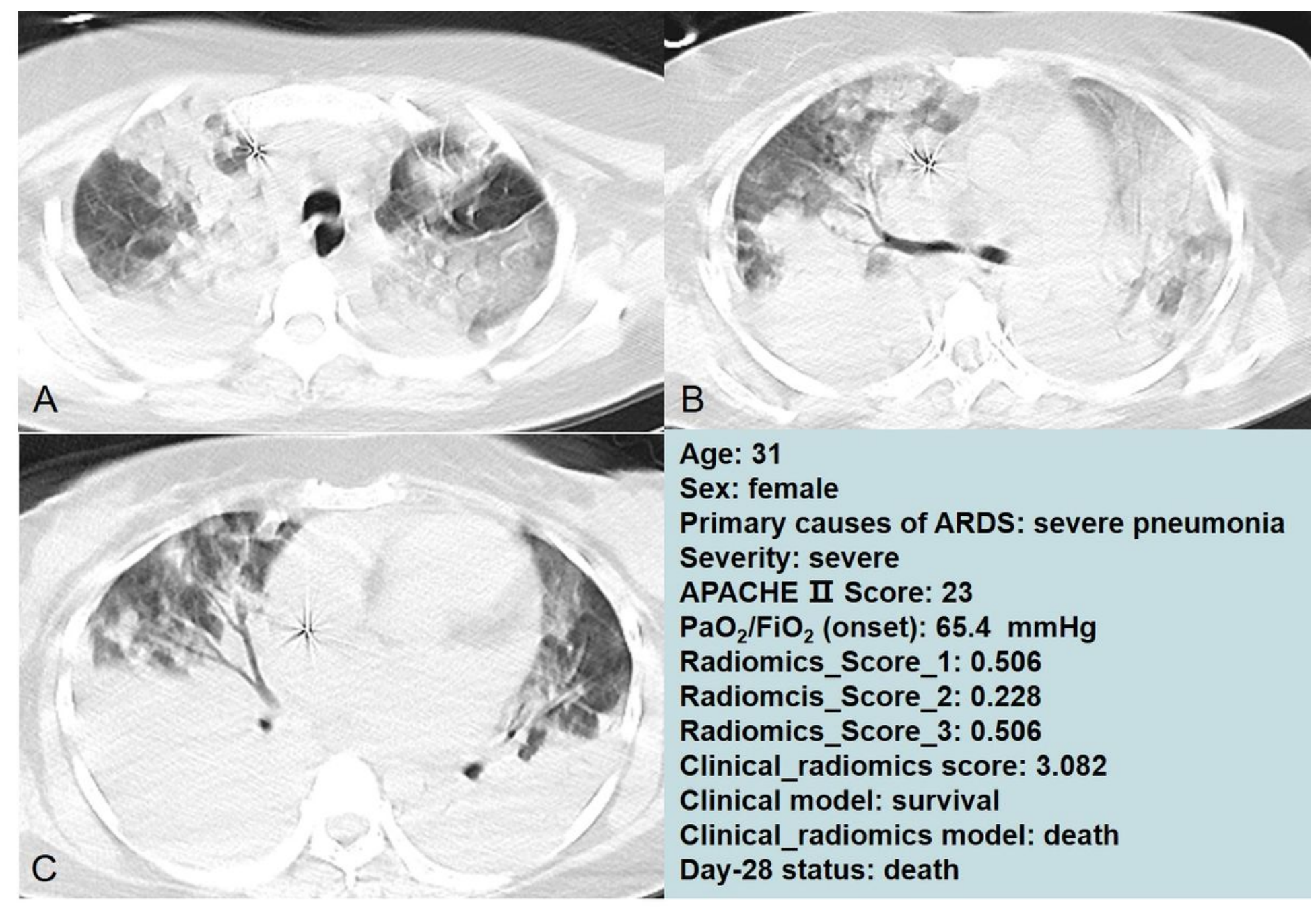

Figure 6

Chest CT images of a 31-year-old female admitted to the intensive care unit for acute respiratory distress syndrome with no special previous history. The CT images were obtained within 24 hours of illness onset. The upper (A), middle (B), and lower (C) lungs show bilateral diffuse opacity. The table show specific metrics. The female died within 28 days of illness onset, and the clinical_radiomics model made the correct prediction.

\section{Supplementary Files}

This is a list of supplementary files associated with this preprint. Click to download.

- supplementalmaterial.docx 